
Y SU CONTRIBUCIÓN AL DESARROLLO ECONÓMICO DE AYANGUE, PROVINCIA DE SANTA ELENA.

Sabina Villón, Brusela Vásquez, Tannia Aguirre. 


\title{
Evolución del sector servicios de alojamiento y restauración y su contribución al desarrollo económico de Ayangue, Provincia de Santa Elena
}

\section{Evolution of the accommodation and restoration services and their contribution to the economic development of Ayangue, province of Santa Elena}

\author{
Sabina Villón Perero, Brusela Vásquez Farfán, Tannia Aguirre Suárez \\ Universidad Estatal Península de Santa Elena \\ e-mail:sabinagisella@hotmail.es
}

\begin{abstract}
Resumen
Las actividades turísticas, en especial los servicios de alojamiento y restauración han mantenido una importante participación en la economía del Ecuador durante los últimos años, a partir de esta premisa se consideró relevante realizar el presente estudio que consiste en el análisis de la evolución del sector en mención y su contribución al desarrollo económico de la comuna Ayangue ubicada en la provincia de Santa Elena. Para ejecutar la investigación de campo se tomó una muestra de establecimientos dedicados a las mencionadas actividades, así como también se realizó un estudio socioeconómico mediante la aplicación de encuestas y entrevistas las mismas que permitieron obtener información cualitativa y cuantitativa, cuyos resultados evidenciaron cómo ha evolucionado este sector y el impacto que ha generado en el desarrollo de la comunidad a través del tiempo, los mismos que reflejaron el incentivo de emprendimientos, la generación de plazas de trabajo y el mejoramiento del nivel económico y social de los comuneros.
\end{abstract}

Palabras clave: Alojamiento, restauración, desarrollo económico, turismo

\begin{abstract}
Tourist activities, specially Accommodation and provision of food and beverages services, have had an important participation in the economy of Ecuador in the last years, based on this premise it was considered relevant to carry out the present study, which is an analysis of the sector in question and their contribution to economic development of the Ayangue Commune, in Province of Santa Elena. For perform field investigations, it took a sample of establishments and was performed a socio-economic study, using surveys and interviews, which they allowed to obtain qualitative and quantitative information, whose results showed the evolution of this sector and the impact generated in the community development through time, resulting incentive enterprises, generating jobs and improving the economic and social level of the community members.
\end{abstract}

Keywords: Accommodation, restoration, economic development, tourism 


\section{Introducción}

El turismo es, sin lugar a dudas, un eje fundamental para la redistribución de la riqueza y la reactivación económica, la generación de empleo, la inversión local y extranjera, el desarrollo de infraestructuras hoteleras, puertos y vías; pero, sobre todo, un atractivo de divisas para el Ecuador. (Clavijo D. Buscán V.,(2012)

La industria de alimentos y bebidas es uno de los sectores más importantes de la economía del país y es una fuente generadora de empleo, por lo tanto su desarrollo permitirá dinamizar la economía, generar valor agregado y responder a las necesidades de empleo. (Fabián Uribe, 2014).

La provincia de Santa Elena, reconocida por sus atractivos y siendo generadora de potencial turístico se encuentra en la franja costera rodeada de un sin número de atractivos entre ellos el balneario Ayangue, una comunidad ubicada en la parroquia Colonche de esta provincia, conocida por su encanto, la piscina natural que en esta se forma y su calidad paisajística conlleva a la visita de propios y extraños, provocando que el turismo en esta localidad desempeñe un rol de gran importancia.

Los servicios turísticos que en esta comunidad se ofertan son cada vez más reconocidos y se debe tomar en cuenta que es de suma importancia conocer la magnitud en la que estas actividades han contribuido a la mencionada comunidad a través del tiempo, con el desarrollo tanto turístico como socioeconómico en dicha comuna, específicamente, porque en la actualidad se desconoce el aporte que este sector productivo brinda al balneario investigado y por ende a la provincia, destacando así el trabajo investigativo y aplicado de manera cuantitativa y cualitativa, el cual ayudará a analizar sobre el desarrollo económico y sustentable que existe en esta localidad y de esta manera observar los resultados acerca del desarrollo económico y turístico que posee esta comuna de la provincia de Santa Elena.

Es menester investigar las condiciones actuales, a través del levantamiento de información, acerca del desarrollo turístico de la Comuna Ayangue, específicamente en la evolución y el impacto socioeconómico que ha generado la oferta de servicios de alojamiento y restauración en la comunidad, durante los últimos años.
La información levantada por estudiantes de la carrera de Gestión y Desarrollo Turístico de la Universidad Península de Santa Elena puede ser de fuente o indicio de nuevas investigaciones o proyectos a realizarse con ayuda de la comunidad y de su mismo desarrollo.

Por tal motivo, se ha planteado la hipótesis de que los establecimientos de alojamiento y restauración contribuyen de manera significativa en el desarrollo socioeconómico de Ayangue.

La investigación se encuentra estructurada de la siguiente manera:

En primera instancia, se relata brevemente la historia de Ayangue, así como también se detalla el desarrollo turístico de la comunidad a través del tiempo, esto comprende desde cómo se inició el turismo hasta los acontecimientos y actividades turísticas que existen actualmente.

Por otra, parte se identificaron las principales actividades turísticas que se practican en el balneario, sus características, los servicios que se ofrecen en cada una de ellas y los paisajes naturales con las que cuenta la localidad.

Los métodos y recursos aplicados constituyeron un valioso instrumento para la obtención de una valiosa información para ser analizada; los resultados del estudio evidenciaron la realidad actual de Ayangue en cuanto al turismo y las actividades que se derivan de ella, haciendo hincapié de la contribución que ha tenido el mencionado sector en el desarrollo del balneario.

\section{Historia y evolución de la comunidad}

Ayangue es un balneario ubicado en la provincia de Santa Elena, cuyos límites son: al norte la comuna San Pedro, al este la parroquia Colonche y al Oeste limita con el Océano Pacífico. Esta localidad es considerada uno de los primeros puertos que tuvieron los Colonchis durante los años comprendidos entre 1811 y 1821 , en este pueblo se encontraban diversidad de especies marinas y esta era la razón principal por la cual era muy visitada. En vista de esto los pobladores de otras comunidades como Colonche, Chanduy y Puerto del Morro consideraron que esta era una oportunidad para generar recursos rentables por medio de la pesca, es así que algunas familias de pueblos aledaños tomaron la decisión de residir en este pequeño recinto, dando inicio a un desarrollo comercial, aproximadamente en el año 1833, el mismo que consistió principalmente en el intercambio de productos pesqueros por agrícolas a manera de trueque. Durante el año 
1952 comenzaron a organizarse como institución comunal pero aún no era considerado un atractivo turístico, tan sólo eran reconocidos como un puerto pesquero y de agricultura, las únicas personas que visitaban este balneario eran los pueblos vecinos y familiares de los residentes, porque no contaba con vías de acceso, la entrada a esta comunidad era a caballo o a pie, fue así donde estas personas comenzaron a difundir que Ayangue contaba con un paisaje natural por su hermosa playa y el mar calmado sin corrientes o resacas, causando un gran impacto a los visitantes y familiares de los habitantes que se quedaban "enamorados" de este bello paraíso. (Archivos de la Casa Comunal)

Las primeras personas en hacer turismo en este balneario fueron motivadas debido a que escuchaban que el mar era como un piscina artificial, fue así que las mujeres de los pescadores comenzaron a implementar pequeños puestos hechos de cuatro estacas y tolda de saco de arroz la cual cubría el fogón de leñas donde se cocinaba el arroz y el pescado frito, brindando un buen servicio al visitante, también comenzaron a realizar paseos en sus pequeños botes donde los turistas conocían y emprendían lo que en ese entonces era la pesca artesanal generándoles ingresos económicos rentables para el desarrollo personal de sus pequeños comedores. (Cobeña D. y Mora R., 2012)

En 1971 se construyó la carretera de acceso principal a esta comunidad, esto incentivó la afluencia de más visitantes y al desarrollo de más servicios de gastronomía, sin dejar de lado a la pesca y al comercio; según archivos que reposan en la Casa Comunal de Ayangue.

Para mejorar la estadía del turista la familia Guale implementó la primera hostería llamada en ese entonces "Un millón de amigos" que actualmente pertenece a otro propietario con el nombre "Hostal Sol y Mar" debido a que el turista visitante solo podía quedarse hasta las 6 de la tarde por la ausencia de hospedaje y servicios básicos que exige el turista, según relató la señora Nelly Maldonado, quien reside en la comuna.

Gracias al desarrollo de esta comunidad el número de ingresos de turistas fue aumentando generando fuentes de trabajo y recursos rentables para los comuneros, además en 1973 obtuvieron el alumbrado público, como poseedores de uno de los servicios básicos principales ya que no todos los sectores de la zona norte de Santa Elena contaban con este servicio, otras de las necesidades básicas de los turistas y comuneros era la comunicación con sus semejantes debido a esto en 1989 se creó la primera cabina telefónica pública para que así tengan un mayor acceso de comunicación hasta que después la comunidad comenzó a tener sus propias líneas.

Los comuneros y turistas demandaban un lugar donde se brinden primeros auxilios y medicina gratuita, como respuesta a esto, en 1990 se fundó el sub-centro de salud, el mismo que actualmente no está implementado correctamente debido a que no cuenta con equipos avanzados, ni una buena infraestructura.

En el 2007 se implementó el servicio de agua potable, los pobladores anteriormente se abastecían del líquido vital por medio de tanqueros pero esta red de agua potable no abastece para toda la comunidad debido al incremento de los moradores, esto constituye un problema serio en épocas de temporada alta, puesto que en hosterías y restaurantes se quedan sin agua. Ayangue de ser solo considerado un recurso natural se transformó en un atractivo turístico respaldado por el Ministerio de Turismo y del Medio Ambiente porque no solamente consta con un balneario de aguas pacíficas si no también con una biodiversidad de flora y fauna, en 2008 se inauguró un parque de recreación. En las temporadas de lluvia la comunidad se inundaba y esto provocaba criaderos de mosquitos, en aquel momento los comuneros solicitaron alcantarillado sanitario, y esto fue respondido inmediatamente y fue inaugurado en el 2010.

Actualmente, la comunidad cuenta con los siguientes negocios que se dedican a brindar servicios turísticos: en hospedaje existen 15 establecimientos, en cuanto a servicios de alimentos están en funcionamiento 71 cabañas comedores, 16 quioscos que ofrecen bebidas tales como cocteles, jugos y batidos, 5 escuelas de buceo, además 47 servidores ambulantes que trabajan solo en temporada alta y otros tipos de negocios que trabajan durante todo el año que comprenden 72 entidades, éstos datos se tomaron de investigaciones realizadas por el doctor Luis Vayas Cevallos.

Las autoridades recientemente han impulsado junto con la directiva el desarrollo de la comunidad cobrando impuestos a todas estas personas que se dedican al turismo. Este ingreso económico que se integra a la comuna sirve como fuentes de ingreso para los individuos que se 
encargan del mantenimiento de la playa, carreteras y parques.

\section{Principales actividades productivas de la Comunidad}

La comuna Ayangue se caracteriza por ser una zona cuyas principales actividades económicas son la pesca y el turismo seguido de las artesanías y agricultura, a pesar de ser una comunidad eminentemente pesquera, un gran porcentaje $(80 \%)$ de la población se beneficia del turismo de manera directa o indirecta.

\section{Pesca}

La actividad pesquera es una de las más importantes en la comuna, ya que existen aproximadamente 400 pescadores artesanales y se cuenta con una flota pesquera de 80 embarcaciones tipo fibras de vidrio. El tamaño promedio de las embarcaciones es de 7,5 metros y el arte de pesca preferiblemente utilizado es el trasmallo. Las pesquerías principales que tiene esta comuna son la del lenguado y langostino. La pesca con arpón es realizada por un pequeño grupo de buzos que efectúan esta actividad utilizando compresores o buceando a pulmón. (relatos de Flavio Salinas, nativo de Ayangue).

\section{Agricultura}

Entre los principales productos que se siembra están la papaya, sandía, melón, zapallo, solo en época de invierno, los mismos que son para el consumo familiar más no para la venta, ya que no cuentan con un sistema de riego.

\section{Turismo}

El turismo es una de las principales actividades que se realiza en la comuna debido a la belleza y tranquilidad de la playa, a su biodiversidad marina costera, y es posible observar diferentes especies como delfines, ballenas, tortugas marinas, spondylus, corales, aves costeras marinas y gran diversidad de peces.

El Islote del Pelado es otro atractivo turístico donde se puede practicar buceo recreativo, snorkel y observación de aves marinas. Durante un recorrido acuático hacia el sitio es posible observar ballenas jorobadas durante la temporada de avistamiento.

La comunidad tiene dos temporadas turísticas bien marcadas: Temporada de playa ciclo costa (marzo- abril) y Temporada de verano ciclo sierra (julio- agosto).

\section{La gastronomía}

Los abuelos de esta comuna narran que en sus inicios Ayangue era una playa virgen al no ser visitada por turistas, solo llegaban familias de los comuneros quienes comentaban a sus amigos y conocidos que visitaran este hermoso balneario que asemeja una piscina pacífica con aguas cristalinas y personas muy hospitalarias.

Cuando empezaron a llegar turistas extranjeros a quienes le gustó mucho por la hermosa playa que vieron, en ese entonces algunos comuneros tomaron la iniciativa de hacer pequeñas cabañascomedor en sus inicios construidas por hojas de palma y estacas, existían en ese entonces 4 comedores donde servían los alimentos a los turistas que llegaban hasta la localidad.

Existían pequeñas balsas donde las personas pescaban a remo, para los distintos comedores de esta zona brindando un producto fresco donde solo los turistas se preocupaban por descansar y disfrutar del buen ambiente, tranquilidad, junto al paisaje maravilloso, a la brisa del mar y a la degustación de la deliciosa gastronomía preparada con los mariscos que esta comunidad ancestral posee.

\section{Deportes acuáticos}

En la comunidad el deporte que surgió inicialmente fue el buceo artesanal por cuanto no se disponía de implementos adecuados para estar debajo del agua optando a mantener la respiración durante un tiempo limitado. Los iniciadores de esta práctica en la comuna fueron los señores Justo Pozo y Don Manuel Pozo hasta que llegó un turista colombiano Diego Arango a quién le fascinó el hermoso paisaje de la Isla del Pelado, este siendo buzo enseñó a los habitantes nuevas técnicas de buceo usando implementos adecuados, pero lastimosamente la población no tomó importancia a lo que decía, después de dos años conoció al señor Ambrosio Yagual a quién contrató para ejercer labores de buceo y gracias a las enseñanzas de este turista don Ambrosio tomó la iniciativa de seguir aprendiendo más acerca de esta práctica; en la actualidad él es un instructor de buceo y tiene su agencia en Ayangue.

Con el paso del tiempo, esta actividad en la comunidad ha ido mejorando y tomando importancia como un deporte marino que ha generado fuentes de trabajo para los pescadores de la comunidad quienes aprovechan a dirigir al turista a la zona de destino donde se encuentra el islote del pelado conocido también como el viejo, este lugar es muy visitado por personas extranjeras y nacionales para practicar este deporte y donde se encuentran diferentes 
especies marinas que habitan en esta reserva, además constituyen un atractivo impresionante las aves que albergan en este parque en donde se puede observar distintas especies de gaviotas, piqueros de patas azules, pelícanos ecuatorianos y peruanos que forman parte de esta atracción. El avistamiento de ballenas en esta comunidad también es una de las actividades que surgen con mayor influencia de visitas turísticas que atraen consigo la danza de las ballenas jorobadas uno de los espectáculos más increíbles que se pueda ver en los meses de junio y septiembre donde los turistas optan por bucear para sentirse conectados con estas especies. (Yela, S.,2011).

\section{Artesanías}

Es una actividad importante en esta comunidad, debido a que se evidencia el arte manual que poseen en la elaboración de redes de pesca en la talla de sus botes, canoas entre otros, también elaboran bisutería donde la materia prima es en su mayoría especies del mar.

Ayangue sin duda es un paraíso que vive del turismo gracias a las actividades que se emplean que son muy positivas en la denominada temporada playera o temporada alta, los comuneros han tenido un ingreso económico que ha sido beneficioso para formar su propia microempresa de hoteles, hostales, restaurantes y escuela de buceos, entre otros.

\section{Materiales y métodos}

Se tomaron como referencia fuentes de información primaria y secundaria que permitieron entender de manera clara la evolución de las actividades que se practican en Ayangue y los determinantes factores que identifican a esta población.

En la investigación documental se reflejó el manejo de documentos redactados por el Cabildo Comunal y archivos que reposan en la Casa Comunal, facilitados por el presidente de la comuna señor Fabián Cochea; permitiendo conocer, comparar y deducir los diferentes enfoques, criterios, conceptualizaciones, análisis, conclusiones y recomendaciones de los autores, con el propósito de ampliar el conocimiento para el trabajo de investigación y obtener información relevante.

El muestreo aplicado fue de tipo no probabilístico, a juicio de las investigadoras, tomando como referencia a 65 establecimientos comprendidos entre servicios de alojamiento y restauración y a 100 hogares. La investigación de campo se realizó utilizando los instrumentos necesarios, mismos que facilitaron la obtención de información histórica y de datos sobre la situación actual de la comuna, para conocer la incidencia de los establecimientos de alojamientos y restauración en el desarrollo socio-económico de Ayangue.

La encuesta y la entrevista fueron los instrumentos aplicados, la primera se aplicó a 15 propietarios de los establecimientos de alojamiento, como hoteles, hostales, hosterías; y a 50 establecimientos de restauración, como restaurantes, barra - bar, cabañas y cafetería, con la finalidad de estudiar variables cuantitativas y cualitativas a través de las preguntas realizadas para que faciliten el proceso de la investigación, por otro, lado la entrevista se realizó al presidente de la comuna como autoridad local, a dos propietarios de las cabañas más antiguas, a un propietario que brinda servicios de alojamiento y a dos nativos de la comuna; a través del cuestionario de preguntas se determinó sus criterios y experiencias adquiridas que aportaron de manera significativa al proyecto de investigación para conseguir información y conocer cuál es la evolución de las actividades turísticas en análisis.

\section{Resultados}

El estudio socioeconómico reflejó que la edad promedio del $70 \%$ de los jefes de hogar es de 35 años, el $100 \%$ de las familias viven en villas propias y el $88 \%$ habitan en estructuras de una planta; para el mayor porcentaje de los pobladores de Ayangue (88\%) el ingreso mensual alcanza hasta un salario equivalente al básico, mientras que el $8 \%$ supera este valor hasta un máximo de $\$ 577$ y el $4 \%$ supera este valor. El $32 \%$ considera que a la comunidad le hace falta mayor promoción turística, seguido del $25 \%$ que manifestó la necesidad de capacitación especialmente en servicios turísticos, el porcentaje restante opinó que la limpieza y la seguridad también son necesidades que deben ser satisfechas en el recinto.

En la actualidad el turismo es considerado como el principal sector que mueve la economía del balneario Ayangue con una participación del $44 \%$, según los resultados de la encuesta realizada a una muestra de 100 familias de la comunidad, sin embargo la pesca aún mantiene un significativo aporte con un $38 \%$, el porcentaje restante corresponde a actividades comerciales como despensas, tiendas, bazares, entre otras. 
Respecto a la parte proporcional dedicada a actividades turísticas el $76 \%$ ofertan servicios de alojamiento y restauración, mientras que la diferencia alquilan carpas, realizan paseos en lanchas, alquilan duchas y servicios higiénicos, entre otras ofertas que se realizan directamente a los turistas que acuden al balneario. Las labores mencionadas generan empleo al $23 \%$ de los hombres y al $85 \%$ de las mujeres.

Para hacer hincapié al objeto de estudio, en cuanto a los servicios de alimentación y hospedaje, se encuestaron a propietarios $y$ administradores de 65 establecimientos, obteniendo como resultado que el $68 \%$ y el $32 \%$ respectivamente es la participación de estos sectores, resaltando así el rol de las mujeres ayanguenses en la preparación y expendio de alimentos.

Por otro lado, se pudo evidenciar que dentro de los establecimientos que ofrecen alojamiento el $67 \%$ son hosterías, el $17 \%$ corresponden a hoteles y el $16 \%$ son hospederías. Mientras que el servicio de alimentos y bebidas está comprendido por el $50 \%$ de restaurantes, el $31 \%$ de barras y bares y el 19\% son cabañas.

El 50\% de los establecimientos de alojamiento tienen hasta 8 años en funciones, seguido del $33 \%$ que llevan en el mercado desde 9 hasta 16 años, mientras tanto el $17 \%$ lleva desde 25 hasta 32 años ofertando el servicio de hospedaje a los turistas que llegan hasta este hermoso balneario. Así mismo, en cuanto a las entidades que ofertan alimentos y bebidas, el mayor porcentaje (44\%) tienen un tiempo máximo de funciones hasta 8 años, seguido del $38 \%$ de establecimientos que han prestado sus servicios desde 9 hasta 40 años, sin embargo se pudo corroborar que el porcentaje restante (18\%) tiene hasta más de 40 años ofertando alimentos y bebidas.. Estos datos evidencian el incremento de establecimientos del mencionado sector en la localidad, a través del tiempo.

La mayor proporción (80\%) de estos establecimientos atienden todos los días, lo cual requiere del talento humano necesario que preste sus servicios a tiempo completo o medio tiempo, sin embargo, en la temporada denominada baja estos establecimientos prescinden de al menos un trabajador de medio tiempo debido a la disminución de afluencia de turistas.

Más del $80 \%$ de los servidores de hospedaje y alimentación manifestaron que las actividades turísticas han contribuido de manera importante al desarrollo socioeconómico de la localidad y que cada día están adoptando nuevas estrategias para que durante todos los meses del año el balneario sea muy concurrido teniendo como consecuencia la generación de empleo y rentabilidad aportando así de manera significativa al progreso de la comunidad entera.

\section{Conclusiones y Recomendaciones}

En la comuna Ayangue el turismo es una actividad que ha evolucionado a través de los años, si bien es cierto hace varios años atrás los pobladores se dedicaban sólo a la pesca, actualmente ofrecen distintos servicios turísticos, los mismos que han mantenido un crecimiento constante convirtiéndose en la principal fuente de ingresos de las familias de esta comunidad, sin embargo es recomendable la ejecución de proyectos de promoción turística que beneficie a la comuna y por consiguiente a la provincia.

Entre las actividades turísticas que se ofertan, es evidente que los servicios de alojamiento y restauración, constituye el sector más productivo con una participación del $76 \%$, no obstante, es indispensable incentivar la oferta de otros servicios, como por ejemplo los deportes que se puedan practicar en este sector y que son atractivos para muchos turistas. Por otro lado, se pudo observar el crecimiento de las actividades en la elaboración de artesanías (bisutería, souvenirs, entre otros), las mismas que son consideradas como oportunidad de trabajo y como generadora de recursos económicos para quienes se dedican a estas labores y de esta manera dinamizar también las actividades turísticas y la economía de Ayangue.

La comuna Ayangue se ha caracterizado por ser una de las más emprendedoras de la provincia de Santa Elena, puesto que ha trabajado de manera organizada y en convenio o asociación con ONG's en la búsqueda de generación de ingresos y oportunidades de trabajo tanto para hombres y mujeres, ya que los hombres se han inclinado a ejercer en el ámbito de las actividades hoteleras y de preparación y venta de bebidas con o sin alcohol, mientras tanto las mujeres se han organizado y crecido en el ámbito gastronómico o de restauración a través de diferentes negocios, restaurantes, cabañas, kioscos, fogones. Es decir que el sector investigado es considerado como fuente generadora de empleo para los comuneros y en mayor proporción para el género femenino.

Es evidente que, con el pasar del tiempo, el servicio de alojamiento ha tenido un incremento considerable, puesto que si hasta los años 80' sólo existían dos establecimientos, actualmente 
los turistas pueden escoger entre 15 entidades donde hospedarse, como hoteles, hostales y hospederías. Numéricamente este crecimiento representa el $66 \%$, considerando el período comprendido desde el 2006 hasta el 2016. Por otro lado la tasa de crecimiento promedio con respecto a los establecimientos de restauración resultó del $42 \%$ por cada 8 años.

\section{Referencias}

Archivos de la Casa Comunal, (2015). Historia de la comuna Ayangue

Clavijo D. Buscán V. (2012). El sector turístico en el Ecuador: Evaluaciòn Macroeconómica y Perspectivas de crecimiento.
Cobeña D.; Mora R. (2012). Desarrollo e implementación de un programa de inocuidad aplicado a servicios de alimentación colectiva en la provincia de Santa Elena comuna Ayangue.

Maldonado Nelly (2015), residente en la comuna, Relatos de la primera hostería de Ayangue.

Salinas Flavio (2015), nativo y pescador de la comuna, Historia de la comuna Ayangue.

Uribe Fabián (2014) Informe sectorial Ecuador: Alimentos.

Vayas Cevallos Luis, (2015). Censo realizado a los emprendimientos en la comuna Ayangue.

Yela, S. (2011). Ayangue costa aventura. Ecuador Costa aventura. 